\title{
ZUR GESCHICHTE DES WORTES
}

\section{SCHMETTERLING.}

In den wörterbüchern von Adelung, Campe, Weigand und Sanders durften sich keine litteraturbelege fur dieses bis jetzt noch wenig aufgeklärte wort finden, die bis an die mitte des vorigen jahrhunderts heranreichen. Sanders bezieht sich auch auf die wörterbücher und sagt, dass schmetterling bei Frisch noch nicht vorkomme, obgleich es als landschaftlich bei Steinbach (1734) erwähnt werde und bei Spate (1691) einen schmïchtigen menschen bezeichne.

Dazu ist folgendes zu bemerken:

1. Was Steinbach und den Spaten betrifft, so ist Sanders angabe correct; bei Frisch ist jedoch das wort auch zu finden:

In seinen 'Deutsch-lat. wörterb. u. s. w. Berlin 1741' s. 208 (anderer teil) heisst es: 'schmettern v. act. eine formierung, welche zugleich ein erzittern andeutet, wie von schutten kommt schuttern, so kommt von schmeten, schmeissen, dieses verbum schmettern. Es hiess vor alters nur stark schmeissen, ohne etwas zu stucken zu zerschmeissen, als Grobian. fol. 174. Welcher krebs keine eyer hat, Den schmetter (schmeisst) wider in die plat (schlissel). Dass es auch nur fur schmeissen und legen genommen worden, s. aus dem wort schmetterling, papilio, der seine eyer auf allerlei grunes schmeisst od. legt, s. schmeissen u. Belgisch smette, macula, smetten maculare, besmetten s. schmitzen.' Auf dieses wort folgt 'zerschmettern'. Unter 'schmeissen' hat Frisch s. 207, sp. 1 folgendes: 'beschmeissen, ovis inquinare carnem vel alias res quae incipiunt corrumpi. Weil man in Niedersachsen sagt smeten fur schmeissen, so ist das wort schmetterling aufgekommen, papilion, weil diese papiliones allerhand kräuter u. blätter mit ihren eyern beschmeissen.' Unter 'papilio' im register der lat. 
wörter hat F.: 'butterfliege, buttervogel, sommervogel, meyenvogel, milchdieb, molkenteller, zelt', also 'schmetterling' nicht, und dies ist die stelle, welche Sanders citiert.

Derselbe Frisch (Johann Leonhard) hat in seinem 'Nouveau Dictionnaire des passagers françois-allemand et allemandfrançois, 1739. Leipzig', in dem deutsch-frz. teile: 'schmetterling m. papillon'. In dem frz.-deutschen teile freilich ist papillon nur' durch 'ein sommer-vogel, ein Zweifalter' widergegeben.

2. Auch vor Steinbach (1734) findet sich das wort:

M. Andreas Stubeln hat in seinem 'Neuen Lateinischen Wörterbuch. Mit Königl. Polnischer u. Churfürstlich Sächsischer Freyheit. Leipzig 1703', im lat.-deutschen teile: 'Papilio, onis m. der schmetterling, das gezelt'. Im deutsch-lat. teile steht 'geschmettert', 'schmetterig', 'zerschmettert', aber 'schmetterling' nicht; dennoch steht 'schmetterling' in register und führt auf das oben erwähnte 'papilio' im lat.-deutschen teile.

Johannes Matthias Krämer hat 'schmetterling' drei mal:

In seinem 'Königl. nider'hoch-teutsch dictionarium oder beider haupt u. grundsprachen wörterbuch, Nürnberg 1719' findet sich im hochd.-niederd. teile: 'schmetterling sommervogel Kapelle, Uiltje, witje'.

In seinem 'Herrlich-grossen teutsch-italiänischen dictionarium .... Nuremberg 1700' hat er: 'schmetterling [dal fiamengo smetten / imbrattare, in tedesco schmitzen / e per consequenza dalla farina di cui imbratta le dita a chi la tocca] farfalla, farfalletta, parpaglione, it [da schmettcrn/tritare] Uovo con la scorza squassata un poco'.

In seinem 'Neuen dictionarium od. wortbuch in teutschitaliänischer sprach' Nurnberg 1678 steht: 'schmetterling m. farfalla, parpaglione v. sommervogel'. Unter 'sommervogel' gibt er 'pfeiff'holder farfalla, farfalletta, parpaglione, volerina' an. In dem noch 2 jabre frtiher, 1676, erschienenen italienischdeutsehen teile ubersetzt er 'parpaglione' mit 'liechtmucke/it. eine pfeiff holter oder sommervogel', 'farfalla (s.711) mit sommervogel, feyfalter', 'farfalletta dim.' mit 'sommervögelein', und 'farfallone' mit 'grosser sommervogel', brauoht also 'schmetterling' nicht.

In 'Andreae Reyheri Lexicon Latino-Germanicum sive 
Theatrum Romano-Teutonicum etc. etc. Lipsiae et Francofurti' 1686 steht: 'papilio, onis, $m$. vermiculus alatus ein sommer-vogel, zwiefalter, schmetterling, molcken-stäuber. Plin. - b. Papilio tentorium etiam est Plin.' Reyherus hat auch im deutschen index: 'schmetterling papilio'.

In dem 'Fons latinitatis bicornis ex optimorum probatissimorumque auctorum philologorum, poetarum, oratorum etc. etc. opus ab Andrea Corvino Oratoriae et linguae latinae quondam in Academia Lipsiensi P. P. inchoatum etc. Francofniti 1653' stelit seite 462: 'Papilio, onis, insectum alatum variorum colorum ein zwiefalter, molckenstehler, molckendieb, schmetterling'; im register: 'schmetterling 462'.

Im 'Vocabularius optimus Gemma dictus qui fere $\overline{\mathbf{l}}$ duobus milibus vocabulis prius neglectis haud modico labore adauctus etc. etc.' (Leipzig anno 1504) steht: 'Papilio est avicula puerorum ein schmetterlinck'.

$\mathrm{Zu}$ avicula puerorum $=$ papilio vgl. Grimm wb. 'feifalter' sp. 1441 mitte und die daselbst angefuhrte stelle in Diefenbach; ausserdem: 'Antea dictus Gemmula-modo vocabulorum Gemma' (anno 1495): 'papilio avicula puerorum etc.'.

3. Zur ermittelung der heimat des wortes mag noch hinzugefugt werden, dass Popowitsch in seinem 'Versuch einer vereinigung der mundarten von Teutschland u. s. w. Wien 1780', seite 515, sagt: 'schmetterling heisst in Sachsen ein bekanntes geschlächt von insecten u. s. w.' und dass er aus anderen landschaften den namen 'schmetterling' nicht anfuhrt.
JENA, den 11. märz 1890.
H. C. BIERWIRTH. 